\title{
Efficacy of Clinical Pathway for the Management of Mucosal Gastric Carcinoma Treated with Endoscopic Submucosal Dissection Using an Insulated-tip Diathermic Knife
}

\author{
Shoji HIRASAKI, Masahito TANIMIZU, Toshikazu MorIwaKI, Ichinosuke HyOdO, \\ Toshiyuki SHINJ**, Norio KOIDE* and Yasushi SHIRATORI**
}

\begin{abstract}
Objective Recently, the cases of early gastric carcinomas which can be treated by endoscopic submucosal dissection (ESD) method have been increasing in our institute. Simple and precise guidelines for treating mucosal gastric carcinoma are necessary for improving the treatment outcome of this disease. In our institute, ESD using an insulated-tip diathermic knife (IT-ESD) was introduced for the treatment of mucosal gastric carcinoma in 1996. The purpose of this study was to evaluate the impact of a clinical pathway and standardize the treatment for mucosal gastric carcinoma treated with ITESD.

Materials and Methods The Clinical Pathway and standardized of treatment for mucosal gastric carcinoma treated with IT-ESD were introduced at our institute in January 2002. We compared the length of hospitalization, total costs, hospital costs, operation time and bleeding rate during the $\mathbf{1 8}$ months before and after January 2002.

Results There was no significant difference in the clinical characteristics of the 20 patients in the control group and the 23 patients in the pathway group. There were 9 and 13 bleeding cases in the respective groups. The mean length of hospitalization, total costs and hospital costs were significantly less for patients in the pathway group. There was no significant difference in the operation time or bleeding rate among the two groups.

Conclusion Our clinical pathway and the standardization of treatment for mucosal gastric carcinoma treated with IT-ESD proved effective for treating patients with mucosal gastric carcinoma and for minimizing
\end{abstract}

the length of hospitalization without compromising patient care.

(Internal Medicine 43: 1120-1125, 2004)

Key words: gastric cancer, clinical pathway, endoscopic mucosal resection, complication

\section{Introduction}

The Clinical Pathway is a financial management system that was developed in the United States under the DiagnosisRelated Group (DRG) and the Prospective Payment Healthcare System (PPS). The Clinical Pathway originally was aimed to shorten the hospital stay and reduce healthcare costs, which has become an increasingly important issue in medicine. In Japan, it is also used for the standardization of medical care and to increase patient satisfaction with medical treatment.

Generally speaking, the Clinical Pathway and standardizing the treatment protocol for gastric carcinoma treated with endoscopic submucosal dissection using an insulated-tip diathermic knife (IT-ESD) is thought to be difficult to adopt because this disease shows various clinical patterns according to the size of tumor (1). The appearance of complications such as bleeding or perforation may further complicate the course of the disease (2), which will result in a longer hospital stay and high hospital costs in some cases. Recent advances in diagnostic techniques have enabled us to identify small and asymptomatic gastric carcinomas, and the cases of early gastric carcinomas that can be treated by endoscopic mucosal resection (EMR) have been increasing. Therefore, a

\footnotetext{
From Department of Internal Medicine, Shikoku Cancer Center, Matsuyama, *Department of Laboratory Medicine and **Department of Medicine and Medical Science, Okayama University Graduate School of Medicine and Dentistry, Okayama

Received for publication December 2, 2003; Accepted for publication June 27, 2004

Reprint requests should be addressed to Dr. Shoji Hirasaki, Shikoku Cancer Center, 13 Horino-uchi, Matsuyama 790-0007
} 


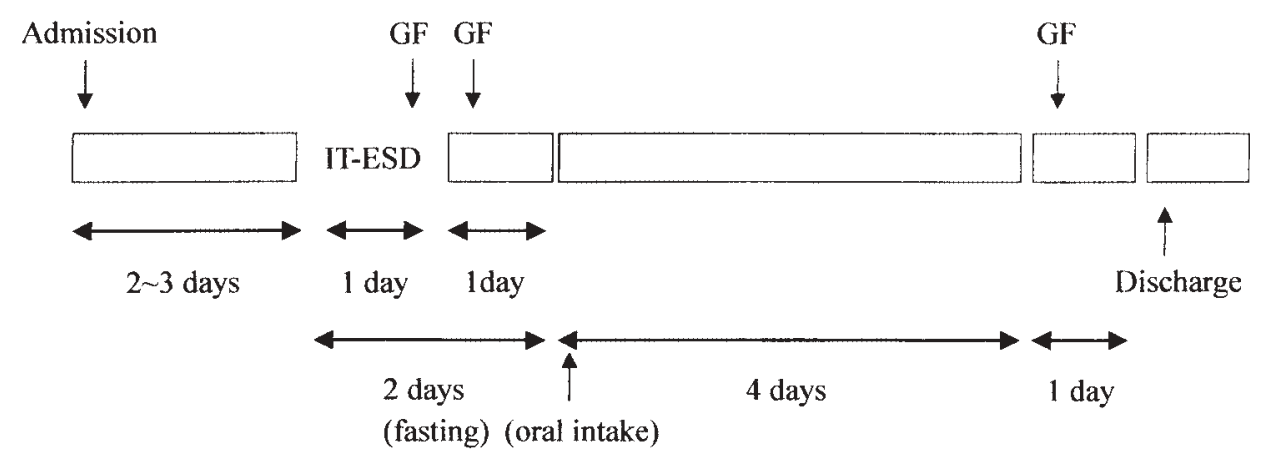

Figure 1. Schema of the clinical course. GF: gastrofiberscope, IT-ESD: endoscopic submucosal dissection using an insulated-tip diathermic knife.

simple and precise medical protocol with a clinical pathway is necessary to improve the treatment outcome and patient satisfaction. In our institute, IT-ESD was applied to patients with early gastric carcinomas up to $20 \mathrm{~mm}$ in diameter that were of a well or moderately histologically differentiated type, and were superficially elevated and/or depressed but without ulceration or definite signs of submucosal invasion. The purpose of this study was to determine the effect of the clinical pathway and standardization of treatment for mucosal gastric carcinoma treated with IT-ESD.

\section{Materials and Methods}

\section{Preoperative assessment}

Until recently, there was no standardized treatment protocol for gastric carcinoma treated with IT-ESD at our institute. Doctors made independent decisions about ordering endoscopic and radiological investigations, diagnosing of mucosal gastric carcinoma, and deciding on the indications for IT-ESD, timing of oral intake, timing of follow-up endoscopy and day of discharge. Until December 2001, patients who were going to undergo IT-ESD were examined by endoscopic ultrasonography (EUS) after admission. And the excision range was also determined after admission. Before the introduction of the clinical pathway, patients were discharged from hospital after meeting all of the following criteria: they could tolerate clear liquids and a regular diet; they had no symptoms due to IT-ESD including epigastric discomfort or abdominal pain; the lapse of 7 to 10 days after ITESD.

We retrospectively reviewed the records of patients admitted to Shikoku Cancer Center with mucosal gastric cancer between October 2000 and December 2001, just before the standardization of treatment with a clinical pathway for mucosal gastric cancer treated with IT-ESD was introduced to our hospital. Since January 2002, all patients admitted for mucosal gastric carcinoma who were going to undergo ITESD have been treated according to our treatment protocol (Fig. 1). The cases associated with other organ carcinoma were excluded from the study. The patients, who had severe underlying disease such as heart disease, respiratory disease, liver disease, or bleeding tendency, were excluded from the indication of pathway. The patients, who had drugs to promote bleeding such as ticlopidine, aspirin or warfarin, were indicated for this pathway after a definite term of discontinuance of drugs. There were no patients who had severe underlying disease or warfarin therapy in the control group and in the pathway group in this study.

All patients fulfilled the following criteria: 1) diagnosed as having mucosal gastric carcinoma by endoscopic findings or EUS, 2) had a biopsy specimen obtained from the lesion that revealed differentiated adenocarcinoma, 3) did not have an ulceration in the lesion, 4) had a tumor up to $20 \mathrm{~mm}$ in diameter. These conditions were confirmed by at least two doctors before IT-ESD. Since January 2002, patients who were going to undergo IT-ESD were examined by EUS before admission. The excision range was decided before admission. They were admitted to our hospital 2 or 3 days before IT-ESD (Fig. 1). Respiratory function and electrocardiogram were checked before IT-ESD. IT-ESD was performed under informed consent.

\section{IT-ESD technique}

IT-ESD was performed as we previously described (3): 1) marks were made at several points along the outline of the lesion with a coagulation current, using a marking tip (Type KD-1L; Olympus) (Fig. 2A), 2) an injection of $20 \mathrm{ml}$ of saline containing $0.0025 \%$ epinephrine was carried out just outside the marks to prevent perforation until the mucosa around the lesion was raised. 3) a hole (about $2 \mathrm{~mm}$ ) for inserting the ceramic ball of the IT-knife into the submucosal layer was made with hot biopsy forceps on the raised mucosa. 4) starting from the hole made by hot biopsy forceps, the mucosa just outside the marks with the IT-knife was incised. 5) after completion of the IT-knife cut around the lesion with a safe lateral margin (Fig. 2B), the submucosal tissue under the circumcised area was abraded with it (Fig. 2C), 6) As the abrasion made progress, the circum- 


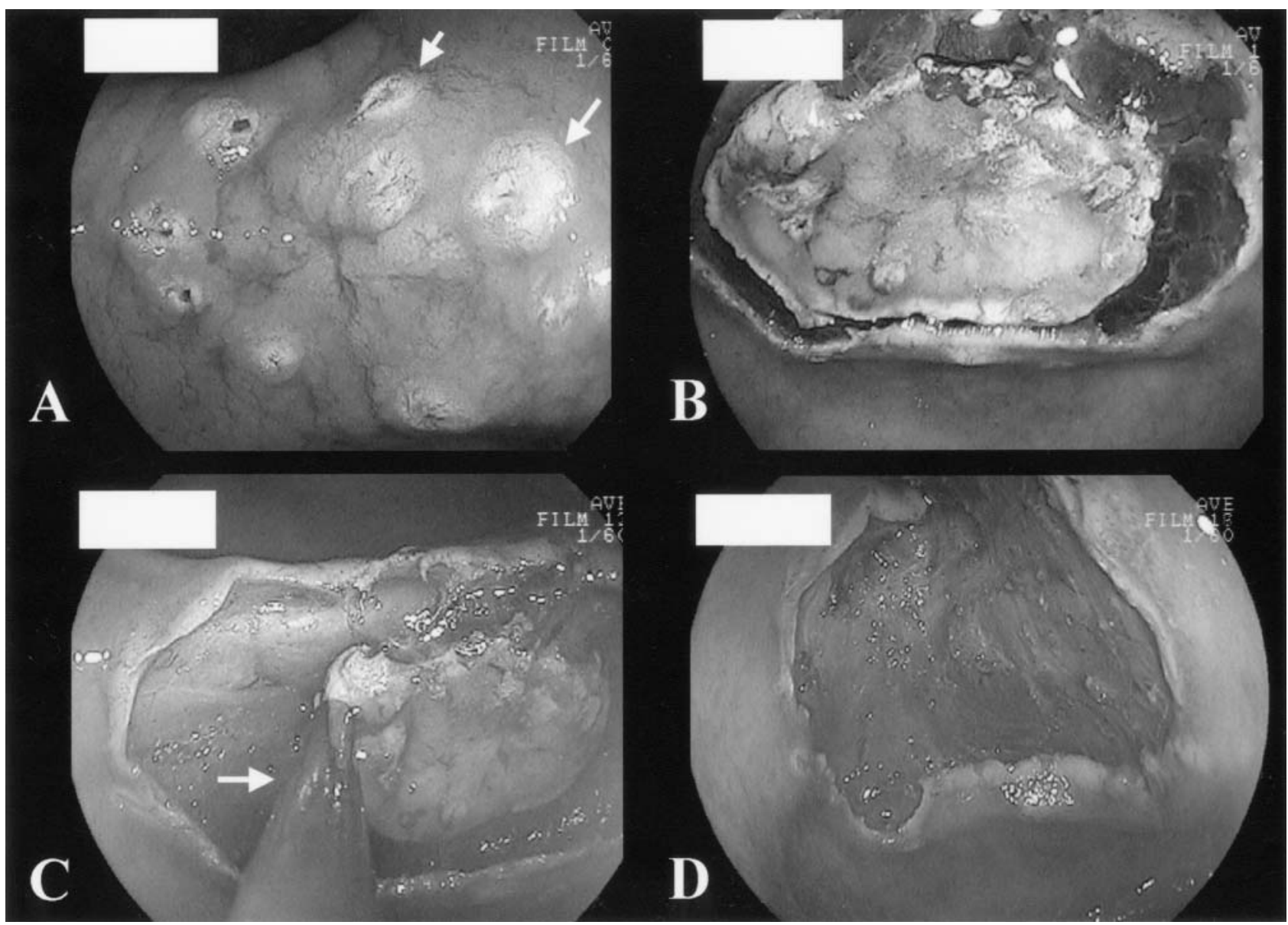

Figure 2. The procedure of IT-ESD; (A) marks (white arrows) are made at several points along the outline of the lesion with a coagulation current. (B) completion of the IT-knife cutting around the lesion with a safe lateral margin. (C) abrasion of the submucosal tissue under the circumcised area with IT-knife (white arrow). (D) the tumor is completely resected.

cised area shrank gradually, 7) an injection of saline with epinephrine was carried out to prevent perforation, until the circumcised area was sufficiently raised to snare the lesion, 8 ) mucosal resection was performed in the same way as with snare polypectomy (Fig. 2D).

\section{Histological assessment}

A gastrointestinal (GI) pathologist evaluated the EMR specimens with special attention to the depth of tumor invasion and the lateral and deep margins of excision. Resected specimens were cut into $2 \mathrm{~mm}$ slices according to the Japanese Classification of Gastric Carcinoma (4) and were evaluated histologically as to whether cancerous glands were present or absent at the margin of each slice.

\section{Post EMR pathway}

All patients were given intravenous proton pump inhibitor (PPI) postoperatively, followed by 4 weeks of oral PPI. Three to five hours after IT-ESD was finished, the first follow-up endoscopic examination was performed to deter- mine whether bleeding would occur or not. The following morning, a second follow-up endoscopic examination was done and oral intake was started on the second postoperative day (Fig. 1). Blood tests were done to determine whether bleeding would occur or not on the next day of IT-ESD. Further examinations were done if necessary on a case-bycase basis. When the patient was free of bleeding or abdominal pain, a third follow-up endoscopic examination after ITESD was performed on the 6th postoperative day (Fig. 1). Patients were discharged from hospital after meeting all of the following criteria: they could tolerate clear liquids and a regular diet; a third endoscopic examination after IT-ESD revealed a decrease in the size of the postoperative ulcer. As a rule, the patients were discharged from hospital on the 7 th postoperative day (Fig. 1).

\section{Patients and methods}

The outcome after introducing this clinical pathway and standardized treatment protocol was investigated. Clinical data were recorded from patients who were admitted to 
Clinical Pathway for IT-ESD

Table 1. Characteristics of Patients and Lesions in the Control Group and the Pathway Group

\begin{tabular}{lccc}
\hline & Control group & Pathway group & P value \\
\hline Number of patients & 20 & 23 & \\
Mean age (years) & $69.8 \pm 7.7$ & $71.1 \pm 5.6$ & NS \\
Male: Female & $15: 5$ & $17: 6$ & NS \\
Size of the lesion $(\mathrm{mm})$ & $11.0 \pm 4.8$ & $9.0 \pm 4.8$ & NS \\
\hline
\end{tabular}

NS: not significant.

Table 2. Results of Clinical Data of Patients in the Control Group and the Pathway Group

\begin{tabular}{lccc}
\hline & Control group & Pathway group & P value \\
\hline Total hospital days & $17.5 \pm 6.9$ & $10.9 \pm 1.9$ & $<0.001$ \\
Preoperative hospital days (days) & $7.2 \pm 5.2$ & $1.9 \pm 0.3$ & $<0.001$ \\
Postperative hospital days (days) & $10.3 \pm 3.0$ & $9.0 \pm 2.6$ & NS \\
Total costs (yen) & $530,000 \pm 106,000$ & $439,000 \pm 75,000$ & $<0.01$ \\
Hospital costs (yen) & $514,000 \pm 117,000$ & $407,000 \pm 76,000$ & $<0.001$ \\
Number of endoscopic examination & $4.3 \pm 1.0$ & $4.2 \pm 0.4$ & NS \\
Operation time (min) & $56.6 \pm 34.7$ & $48.4 \pm 25.7$ & NS \\
Size of the resected specimen (mm) & $30.9 \pm 9.0$ & $28.7 \pm 7.8$ & NS \\
Complications & 45 & 56 & NS \\
Rate of bleeding (\%) & 0 & 0 & \\
Rate of perforation $(\%)$ & & & \\
\hline
\end{tabular}

NS: not significant.

Shikoku Cancer Center for mucosal gastric carcinoma between January 2002 and March 2003. The results were compared with the historical data of patients admitted to Shikoku Cancer Center for mucosal gastric carcinoma between October 2000 and December 2001, and were cared for in a different manner, not according to the pathway.

Patients in both periods were divided into a bleeding group and a non-bleeding group. The bleeding group was defined as patients who required endoscopic management by using methods such as clip placement and/or monopolar electrocoagulation to stop the bleeding. The total length of hospitalization, preoperative stay, postoperative stay, hospital costs, total costs (from the point of the diagnosis of gastric carcinoma to the point of discharge), the number of endoscopic examination (from the point of the diagnosis of gastric carcinoma to the point of discharge, excluding the point of IT-ESD), and operation times were compared among the groups. Patients, who were discharged from hospital on the 8th postoperative day or later, were judged as variance.

\section{Statistics}

Values are expressed as mean \pm SD. Statistical analysis was performed using the unpaired Student's t-test. A P value of less than 0.01 was considered to be significant.

\section{Results}

There were 21 patients in the control group and 24 in the pathway group. One patient in the control group and one patient in the pathway group were excluded from the study as they were associated with rectal cancer and underwent surgical treatment for rectal cancer after IT-ESD during the same hospitalization. All lesions in this study were resected as a single fragment. The diagnosis of mucosal gastric carcinoma was confirmed histopathologically in all patients who underwent IT-ESD.

The number of patients classified in each group and the clinical data of IT-ESD are shown in Table 1. There were no significant differences in any items tested between the two groups. The mean age of the 43 patients was $70.5 \pm 6.7$ years (range, 48 to 84 years), being $69.8 \pm 7.7$ years in the control group and $71.1 \pm 5.6$ years in the pathway group. The size of the lesion was $9.9 \pm 4.8 \mathrm{~mm}$ (range, 2 to $19 \mathrm{~mm}$ ), being $11.0 \pm$ $4.8 \mathrm{~mm}$ in the control group and $9.0 \pm 4.8 \mathrm{~mm}$ in the pathway group.

The data that should be compared between each group are shown in Table 2. There were no significant differences in the mean size of the resected specimen among the groups (30.9 \pm 9.0 in the control group versus $28.7 \pm 7.8$ in the pathway group). The mean length of hospitalization became significantly shorter in the pathway group $(17.5 \pm 6.9$ in the control group versus $10.9 \pm 1.9$ in the pathway group) $(\mathrm{p}<0.001)$. The mean preoperative stay was significantly 
HIRASAKI et al

Table 3. Effect of Bleeding upon Total Hospital Days, Postoperative Hospital Days and Hospital Costs

\begin{tabular}{|c|c|c|c|c|c|c|}
\hline & \multicolumn{2}{|c|}{ Control group } & \multicolumn{4}{|c|}{ Pathway group } \\
\hline & Bleeding & Non-bleeding & & Bleeding & Non-bleeding & \\
\hline Number of patients & 9 & 11 & & 13 & 10 & \\
\hline Size of the lesion $(\mathrm{mm})$ & $12.3 \pm 4.8$ & $9.8 \pm 4.5$ & NS & $9.2 \pm 4.6$ & $8.6 \pm 4.7$ & NS \\
\hline Size of the resected specimen $(\mathrm{mm})$ & $33.3 \pm 9.8$ & $28.9 \pm 7.2$ & NS & $31.2 \pm 6.1$ & $25.4 \pm 8.6$ & NS \\
\hline Total hospital days & $18.7 \pm 8.1$ & $16.5 \pm 5.5$ & NS & $13.9 \pm 2.8$ & $12.4 \pm 2.5$ & NS \\
\hline Postperative hospital days & $12.0 \pm 2.9$ & $8.7 \pm 2.3$ & NS & $10.2 \pm 2.7$ & $8.0 \pm 0$ & NS \\
\hline Hospital costs & $533,000 \pm 126,000$ & $392,000 \pm 133,000$ & NS & $433,000 \pm 64,000$ & $394,000 \pm 83,000$ & NS \\
\hline
\end{tabular}

shorter in the pathway group $(7.2 \pm 5.2$ in the control group versus $1.9 \pm 0.3$ in the pathway group) $(\mathrm{p}<0.001)$. There were no significant differences in the mean postoperative stay among the groups $(10.0 \pm 3.0$ in the control group versus $9.0 \pm$ 2.6 in the pathway group). The mean hospital cost in Japanese yen became significantly lower in the pathway group $(514,000 \pm 117,000$ in the control group versus $407,000 \pm 76,000$ in the pathway group) $(\mathrm{p}<0.001)$. The mean total cost became significantly lower in the pathway group $(530,000 \pm 106,000$ in the control group versus $439,000 \pm$ 75,000 in the pathway group) $(\mathrm{p}<0.01)$. There was no significant difference in the number of endoscopic examinations among the groups $(4.3 \pm 1.0$ in the control group versus $4.2 \pm$ 0.4 in the pathway group). In the control group, all patients underwent endoscopic examinations at least 2 times namely, 1) at the point of diagnosis of gastric cancer and 2) on the day following IT-ESD. One patient underwent 6 endoscopic examinations: 1) at the point of diagnosis of gastric cancer, 2) at the point of EUS in admission, 3) five hours after ITESD 4) emergency endoscopy due to bleeding after IT-ESD in the night, 5) on the day following IT-ESD, and 6) on the 6th postoperative day. This was the case that underwent the most endoscopic examinations in the control group. In the pathway group, 19 of 23 patients underwent endoscopic examinations 4 times (once at the point of diagnosis of gastric cancer, 3 times according to treatment protocol). The remaining 4 patients underwent endoscopic examinations 5 times, one underwent an additional endoscopic examination due to bleeding after IT-ESD and 3 patients underwent an additional endoscopic examination due to EUS. The mean operation time of the 43 patients was $52.2 \pm 28.8$ minutes (range, 15 to 120 minutes), being $56.6 \pm 34.7$ minutes for the control group and $48.4 \pm 25.7$ minutes (range, 15 to $120 \mathrm{~min}$ utes) for the pathway group. There were $22(51.2 \%)$ cases of bleeding during or after IT-ESD: $9(45.0 \%)$ in the control group and $13(56.5 \%)$ in the pathway group. No patients required surgery, angiography or transfusion for bleeding. Perforation did not occur in any patient.

Effect of bleeding upon total hospital days, postoperative hospital days and hospital costs was shown in Table 3. There was no significant difference in the total hospital days, postoperative hospital days or hospital costs between the bleeding group and non-bleeding group in the control group and the pathway group (Table 3 ).

Five patients did not follow this pathway in the pathway group, and were discharged from the hospital on the 8th postoperative day or later, resulting in a variance rate of $21.7 \%$. In all 5 cases, the start of diet was delayed due to bleeding.

\section{Discussion}

The Clinical Pathway has become widely adopted in Japan since its introduction by the DRG and PPS, and has been under trial in several hospitals since 1998 (5). In making a clinical pathway, the treatment of a particular disease needs to be standardized, but since this is a standard scale, some cases do not follow a predictable course and variance from the pathway is inevitable. Mucosal gastric carcinoma treated with IT-ESD shows various clinical patterns according to the size of tumor or the degree of bleeding, so variance from the pathway is a major problem that needs to be resolved.

Although the mean length of hospitalization was reduced after the introduction of this clinical pathway, there was no significant difference in the mean postoperative stay between the control group and the pathway group. The reduction of the mean length of hospitalization was due to shortening of the preoperative stay. This result has been realized due to the stipulation to confirm the depth of the tumor before admission. We were able to perform IT-ESD in a few days after admission because two or more endoscopists confirmed the depth of tumor or excision range before admission.

We classified cases of mucosal gastric carcinoma treated with IT-ESD into two groups, according to whether or not there was bleeding during IT-ESD or after. Because a bleeding case differs from a non-bleeding case in its endoscopic treatment and use of clips, it is associated with a longer postoperative stay and higher hospital costs. But there were no significant differences in the mean postoperative stay or hospital costs among the bleeding group and non-bleeding group. These results indicate that even patients with bleeding were treated without delay, although bleeding may complicate the course of administration. However, 5 patients did not follow this pathway, resulting in a variance rate of $21.7 \%$ in this study. In all 5 cases, the start of the diet was delayed 


\section{Clinical Pathway for IT-ESD}

due to bleeding. We thought that the variance rate of $21.7 \%$ in this study was tolerated because nearly $80 \%$ of the patients followed the treatment protocol. It has been described that ordinarily about $20 \%$ patients do not follow the Clinical Pathway (6). The reported incidence of bleeding in IT-ESD was higher than in EMR using conventional methods. Ohkuwa et al (1) reported that the incidence of bleeding in IT-ESD was as high as $22 \%$. In our study, the bleeding frequency was higher than Ohkuwa et al (1) reported. Thus, variant cases may decrease in the future, if the bleeding in IT-ESD is well controlled. Improved devices for the management of bleeding are certainly necessary to decrease the length of hospitalization. There were no perforation cases in this study. However, it is necessary to pay particular attention to perforations because Ohkuwa et al (1) and Ono et al (7) reported that the incidence of perforation with IT-ESD is $5 \%$.

In the present study, operation time with IT-ESD was longer than that of EMR using conventional methods. Although the IT-ESD technique is complicated, it might be worth trying as the first therapy for mucosal gastric carcinoma because of its high one-piece resection rate $(1,2)$. The introduction of our treatment protocol did not affect the operation time of IT-ESD.

We decided that patients underwent 3 times of follow up endoscopy in the treatment protocol. Before introduction of the clinical pathway, all patients in the control group underwent a follow-up endoscopic examination the following morning. We create the first follow-up endoscopic examination to treat with bleeding soon after IT-ESD and the third follow-up endoscopic examination to confirm the improving of the postoperative ulcer. The result was that the total number of endoscopy in the pathway group did not increase compared with control group. We speculated that the creation of 3 times of follow up endoscopy in the treatment protocol did not affect the total costs or hospital costs.

We think that it is possible to hospitalize patients on the day before IT-ESD if the preoperative examination is finished as an outpatient. Consequently we can shorten preoperative hospital days and total hospital days. Now we are putting this idea into practice. In the pathway group, moreover, when bleeding did not occur at the second follow-up endoscopic examination, there was no GI bleeding thereafter. Thus, we are considering a new pathway in which the third follow-up endoscopic examination after IT-ESD is omitted and the patients are discharged from hospital on the 6th postoperative day.

By making the treatment protocol and criteria for discharge clear, patients and nurses were able to understand the treatment more clearly, which helped them to accept it. As a result, our clinical pathway and standardization of treatment for mucosal gastric carcinoma improved the satisfaction of both patients and nurses, being well accepted by both. Reducing hospital costs is of paramount importance because the number of cases of early gastric carcinomas that can be treated by EMR has been increasing at our institute every year. A simple and precise clinical pathway and medical guidelines for treating mucosal gastric carcinoma would have a great impact on hospitals, and promote patient satisfaction.

Our clinical pathway and standardization of treatment for IT-ESD for mucosal gastric carcinoma decreased the total length of hospitalization and overall healthcare costs. These results suggest that our clinical pathway and standardization of treatment for IT-ESD for mucosal gastric carcinoma had a successful outcome and promoted the quality of care.

\section{References}

1) Ohkuwa M, Hosokawa K, Boku N, Ohtu A, Tajiri H, Yoshida S. New endoscopic treatment for intramucosal gastric tumors using an insulated-tip diathermic knife. Endoscopy 33: 221-226, 2001.

2) Miyamoto $S$, Muto $M$, Hamamoto $Y$, et al. A new technique for endoscopic mucosal resection with an insulated-tip electrosurgical knife improves the completeness of resection of intramucosal gastric neoplasms. Gastrointest Endosc 55: 576-581, 2002.

3) Hirasaki S, Endo H, Nishina T, Masumoto T, Tanimizu M, Hyodo I. Gastric cancer concomitant with inflammatory fibroid polyp treated with endoscopic mucosal resection using an insulation-tip diathermic knife. Intern Med 42: 259-262, 2003.

4) Japanese Research Society for Gastric Cancer. Japanese classification of gastric carcinoma. Kanehara \& Co., Ltd., Tokyo, 1999.

5) Takegami K, Kawaguchi Y, Nakayama H, Kubota Y, Nagawa H. Impact of a clinical pathway and standardization of treatment for acute appendicitis. Surg Today 33: 336-341, 2003.

6) Dykes PC. Desining and implementing clitical pathways: An overview. in: Dykes PC, Wheeler K (Eds). Planning, Implementing and Evaluating Clitical Pathways. Springer Publishing Company, New York, 1997.

7) Ono H, Kondo H, Gotoda T, et al. Endoscopic mucosal resection for treatment of early gastric caner. Gut 48: 225-229, 2001. 